\title{
sciendo
}

Int. J. of Applied Mechanics and Engineering, 2018, vol.23, No.4, pp.835-853

DOI: 10.2478/ijame-2018-0046

\section{CALCULATION METHOD FOR PLATES WITH DISCRETE VARIABLE THICKNESS UNDER UNIFORM LOADING OR HYDROSTATIC PRESSURE}

\author{
G. ARYASSOV, D. GORNOSTAJEV and I. PENKOV* \\ Department of Mechanical and Industrial Engineering, School of Engineering \\ Tallinn University of Technology \\ Ehitajate tee 5, 19086 Tallinn, ESTONIA \\ E-mail: igor.penkov@ttu.ee
}

\begin{abstract}
The article proposes a new analytical method for the calculation of plates with constant and variable rigidity parameters. This method renders it possible to decrease the weight of the plates working under hydrostatic pressure by using variable thicknesses.

Firs, a short overview of existing calculation methods and their results are compared. It is shown that all existing methods depend on boundary conditions. Then is given the theory of the proposed calculation method is described and calculations for plates with constant and variable thickness worked under uniformly loaded forces and hydrostatic pressure are made. The results are compared to the FEM calculations and experimental results obtained by a tensile test machine and special equipment. Calculation results obtained by the proposed analytical method and FEM results are very close. Deviations are not more than $11 \%$. Deviations between theoretical calculations and experimental results depend on loading type and design of the test specimens but maximum values are not more than $17 \%$.

The proposed calculation method does not depend on the boundary conditions and can be used for plate calculations. Especially for plates with difficult design and complex loading.
\end{abstract}

Key words: plate calculation, deflection, hydrostatic pressure, boundary conditions

\section{Introduction}

Thin plates are initially flat structural members bounded by two parallel planes called faces and a cylindrical surface (round plates) or two pairs of parallel planes called edges or boundaries. The boundaries are usually perpendicular to the plane faces. The distance between the plane faces is called the thickness of the plate and it is small in comparison with other characteristic dimensions of the plates (length, width, diameter, etc.). Geometrically, plates are bounded either by straight or curved boundaries. The static or dynamic loads carried by plates are predominantly perpendicular to the plate faces [1]-[6].

The theoretical tasks of the calculations of plate bending even for simple shape plates with constant thickness face some mathematical problems, which in most cases can be solved by approximation methods or by numerical methods. The mathematical problems are growing if the plate has variable rigidity. For those cases theoretical solutions are provided in general for round and rectangular plates with linear thickness changing [7]-[14].

Real solutions of plate calculations can be provided only in some particular cases, mainly for plates with constant thickness, simple shape and with special boundary conditions [15]. Variational methods of calculations are one of the more efficient instruments for defining deflections of plates and other components in more difficult cases [16]-[19]. In this research work, we propose an analytical method for the calculation of stress and deformations in rectangular plates with discrete variable thickness. The method can be used for plates under uniformly distributed loads and for plates under hydrostatic pressure.

\footnotetext{
* To whom correspondence should be addressed
} 


\section{Overview and analysis of existing calculation methods}

Basic calculation methods are described in many teaching books and monographs [16]-[18]. Main of them are:
a) Navier' method,
b) Levy solution,
c) collocation method,
d) Kantorovits-Vlassov method,
e) grid method,
f) plate calculations by FEM. be made.

The last three methods will be briefly described in this paper and an analysis of calculation results will

\subsection{Kantorovits-Vlassov method for a four side fixed plate}

The Kantorovits-Vlassov [16]-[18] method is one of the approximation methods. The plate fixed on four sides with the relation $b / a=1$ with a distributed load is shown in Fig.1.

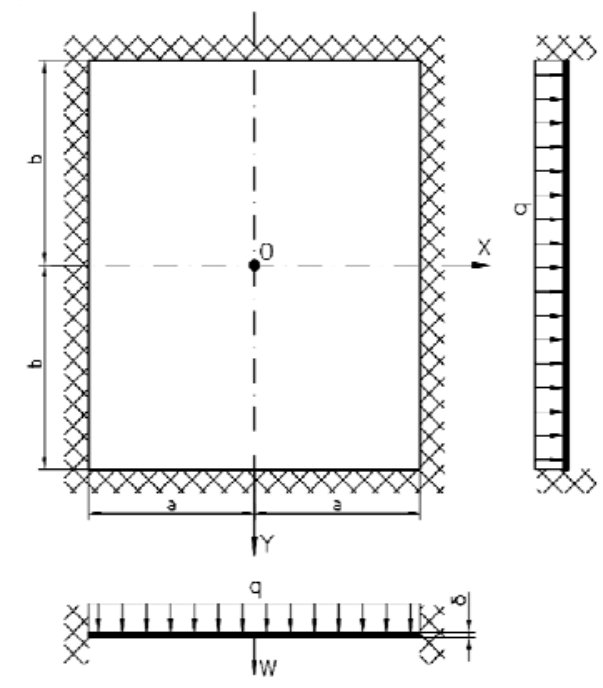

Fig.1. Plate fixed on four sides under an uniformly distributed load.

The boundary conditions are

$$
(w)_{x=a}=0 ; \quad\left(\frac{\partial^{2} w}{\partial x^{2}}\right)_{x=a}=0 .
$$

The equation for deflection is defined by

$$
w=Y(y)\left(1-\xi^{2}\right)^{2}
$$

where $\xi=x / a$. 
To satisfy the boundary conditions on the sides $x= \pm a$, the differential equation of deflection can be presented as follows

$$
\nabla^{2} \nabla^{2} w-\frac{q}{D}=0
$$

After all the calculations [21] we can be obtain

$$
w=\left(\begin{array}{l}
-0.014 \operatorname{ch} \frac{2,075 y}{a} \cos 1.143 \frac{y}{a}- \\
-0.004 \operatorname{sh} \frac{2,075 y}{a} \sin 1.143 \frac{y}{a}+\frac{1}{24}
\end{array}\right) \cdot\left(1-2 \frac{x^{2}}{a^{2}}+\frac{x^{4}}{a^{4}}\right) \frac{q a^{4}}{D} .
$$

The deviation is for the shearing forces up to $14 \%$ from the real magnitudes and for the vertical reactions up to $15 \%$. The Kantorovits-Vlassov method can give deviation equal to zero for the calculation of deflection and for the calculation of the bending moment the deviation can be $0.5 \%$ [16]. Figure 2 shows the deviation between the Kantorovits-Vlassov method and the real values for uniformly distributed load (Fig.2a) and for the hydrostatic pressure (Fig.2b).
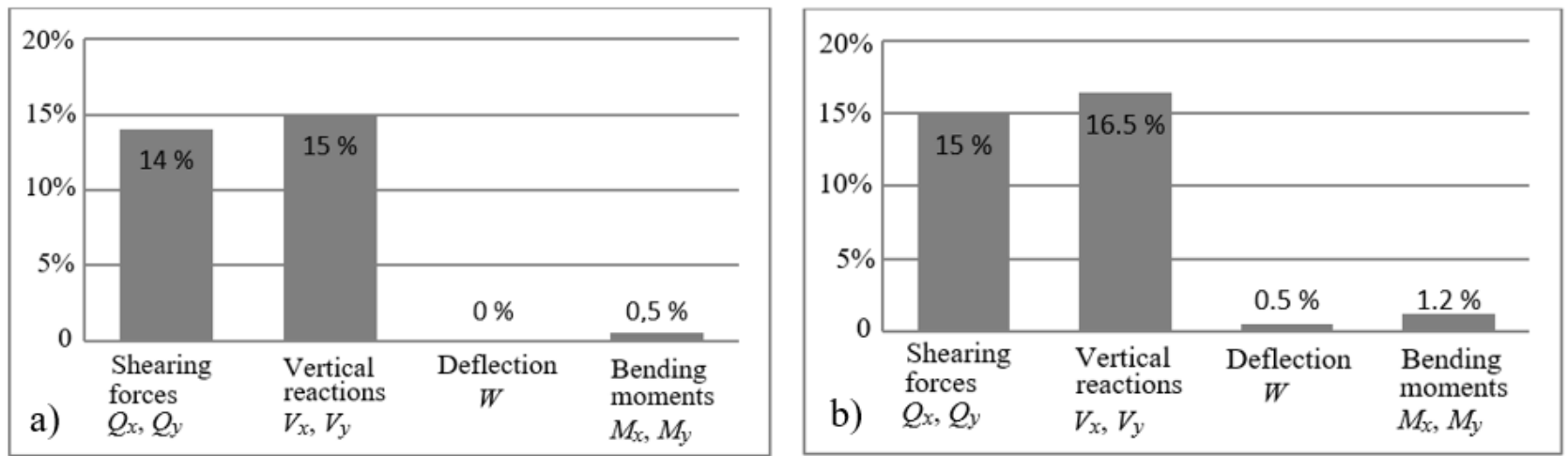

Fig.2. Deviation between the Kantorovits-Vlassov method and the real values for the uniform load distribution (a) and for the hydrostatic pressure (b).

As it can be seen in Fig.2, the Kantorovits-Vlassov method has a small deviation from the real values for the deflection $W$ and for the bending moments $M$ and can be used in plate calculations taking into account the deviation for shearing forces $Q$ and vertical reactions $V$ that can be up to $16.5 \%$. The deviation for the hydrostatic pressure is on the same level as for the uniformly distributed load.

\subsection{Grid method for the plate calculation}

Let us divide the plate into a mesh as shown in Fig.3 [16]; the step in the direction of the axis $X$ is $\Delta_{x}$, in the direction of axis $Y$ it is $\Delta_{y}$. Mesh points around the point $C C$ are as shown in the figure.

The first derivative in the point $C C$ can be defined by

$$
\left(\frac{\partial w}{\partial x}\right)_{c c}=\frac{-w_{b c}+w_{d c}}{2 \Delta_{x}}
$$


The derivative with two variables is defined by

$$
\left(\frac{\partial^{4} w}{\partial x^{2} \partial y^{2}}\right)_{c c}=\left[\frac{\partial^{2}}{\partial y^{2}}\left(\frac{\partial^{2} w}{\partial x^{2}}\right)\right]_{c c}=\frac{\left(\frac{\partial^{2} w}{\partial x^{2}}\right)_{c b}-2\left(\frac{\partial^{2} w}{\partial x^{2}}\right)_{c c}+\left(\frac{\partial^{2} w}{\partial x^{2}}\right)_{c d}}{\Delta_{y^{2}}}
$$

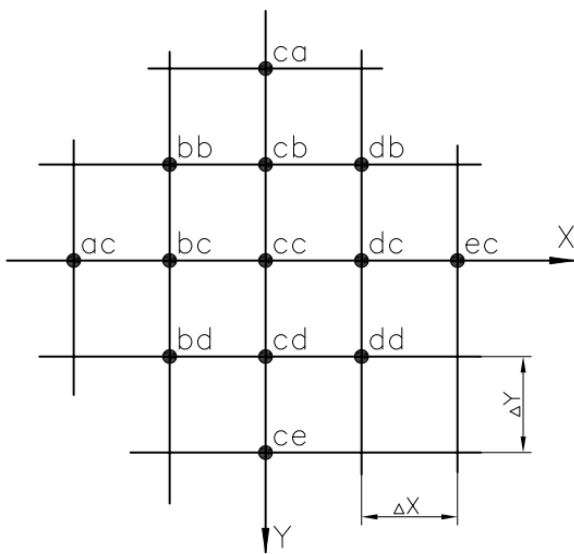

Fig.3. Plate divided by a mesh.

After some calculations a harmonized equation can be obtained

$$
\frac{\partial^{4} w}{\partial x^{4}}+2 \frac{\partial^{4} w}{\partial x^{2} \partial y^{2}}+\frac{\partial^{4} w}{\partial y^{4}}=\frac{q}{D}
$$

Under the consideration that

$$
\left(\frac{\Delta_{y}}{\Delta_{x}}\right)^{2}=\alpha
$$

For a plate fixed in all four edges and with the boundary condition $w_{c c}=0$ we can be obtain

$$
\left(\frac{\partial w}{\partial x}\right)_{c c}=\frac{-w_{b c}+w_{d c}}{2 \Delta_{x}}=0
$$

After simplification and taking into account the boundary condition, for a free landed plate we can obtain

$$
\left(\frac{\partial^{2} w}{\partial x^{2}}\right)_{c c}=\frac{w_{b c}-2 w_{c c}+w_{d c}}{\Delta_{x^{2}}}
$$

Figure 4 demonstrates the deviation between the results obtained by the grid method [22-28] and the real values for the uniformly distributed load (Fig.4a) and for hydrostatic pressure (Fig.4b). 
As can be seen from Fig.4, the grid method has a small deviation from the real values for the deflection $W$ and can be used in the plate calculations taking into account the major deviation from the real values for the shearing forces $Q$ and the vertical reactions $V$.
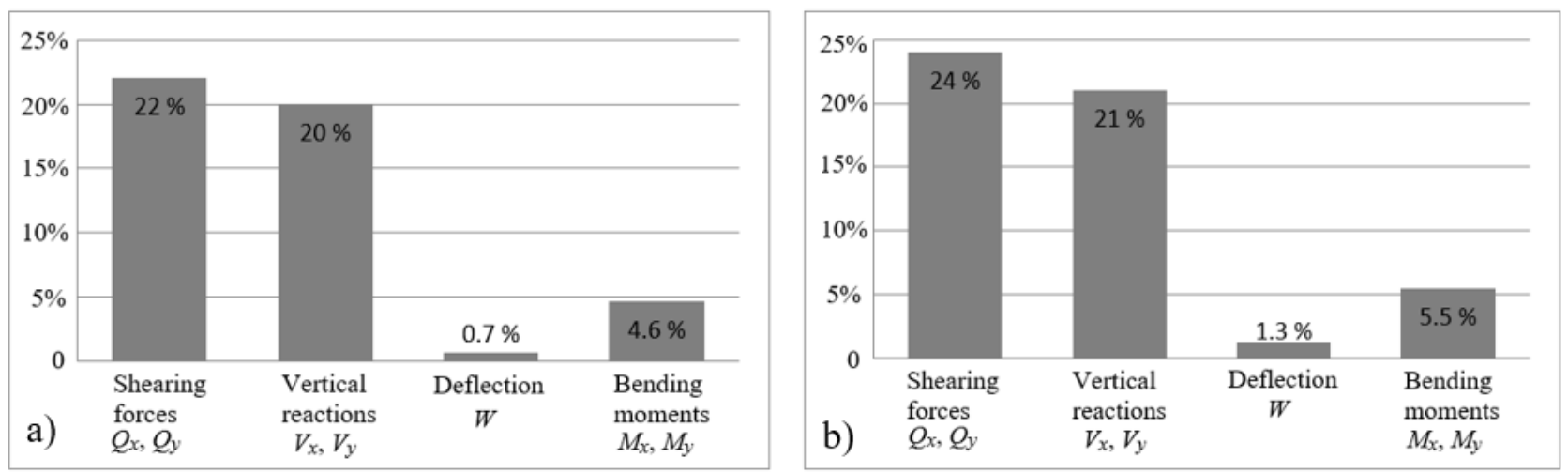

Fig.4. Deviation between the grid method and the real values for the uniform load distribution (a) and for the hydrostatic pressure (b).

\subsection{Plate calculations by FEM}

Calculations for the plate can be provided by the integration of the biharmonic equation, which consists of the derivative of the plate deflection perpendicular to the plate plane. Under finite elements, deflection $w$ can be defined by using an equation that has a matrix $[N]$ and an angular displacement $\{\delta\}^{e}$. Usually, it is used for rectangular and triangular elements in the plate calculations. In each node $n$ the movement has three components such as the deflection that is perpendicular to the plate plane $w$ in the direction of axis $z$, the rotation angle $\left(\theta_{x}\right)_{n}$ around the axis $x$ and the rotation angle $\left(\theta_{y}\right)_{n}$ around the axis $y$. follows

There are twelve parameters for each element, so for the deflection $w$ a polynomial can be written as

$$
\begin{aligned}
& w=\alpha_{1}+\alpha_{2} x+\alpha_{3} y+\alpha_{4} x^{2}+\alpha_{5} x y+\alpha_{6} y^{2}+\alpha_{7} x^{3}+ \\
& +\alpha_{8} x^{2} y+\alpha_{9} x y^{2}+\alpha_{10} y^{3}+\alpha_{11} x^{3} y+\alpha_{12} x y^{3}
\end{aligned}
$$

Constants $\alpha_{1}, \alpha_{2}, \ldots, \alpha_{12}$ can be defined from the equations system.

In the matrix it can be written as follows

$$
\{\delta\}^{e}=[C]\{\alpha\}, \quad \text { where } \quad\{\alpha\}=[C]^{-1}\{\delta\}^{e}
$$

The deflections inside the elements can be defined by

$$
\{w\}=[N]\{\delta\}^{e}=[P][C]^{-1}\{\delta\}^{e}
$$

Figure 5 demonstrates the deviation between the results obtained by the FEM [29-40] and the real values for the uniformly distributed load (Fig.5a) and for hydrostatic pressure (Fig.5b). 
As shown by the figure, the FEM has a large deviation from the real values for the deflection $W$, bending moments $M$, shearing forces $Q$ and vertical reactions $V$. Despite this and keeping in mind that other method are not suitable for comparing plate deflections, the FEM will be preferred.
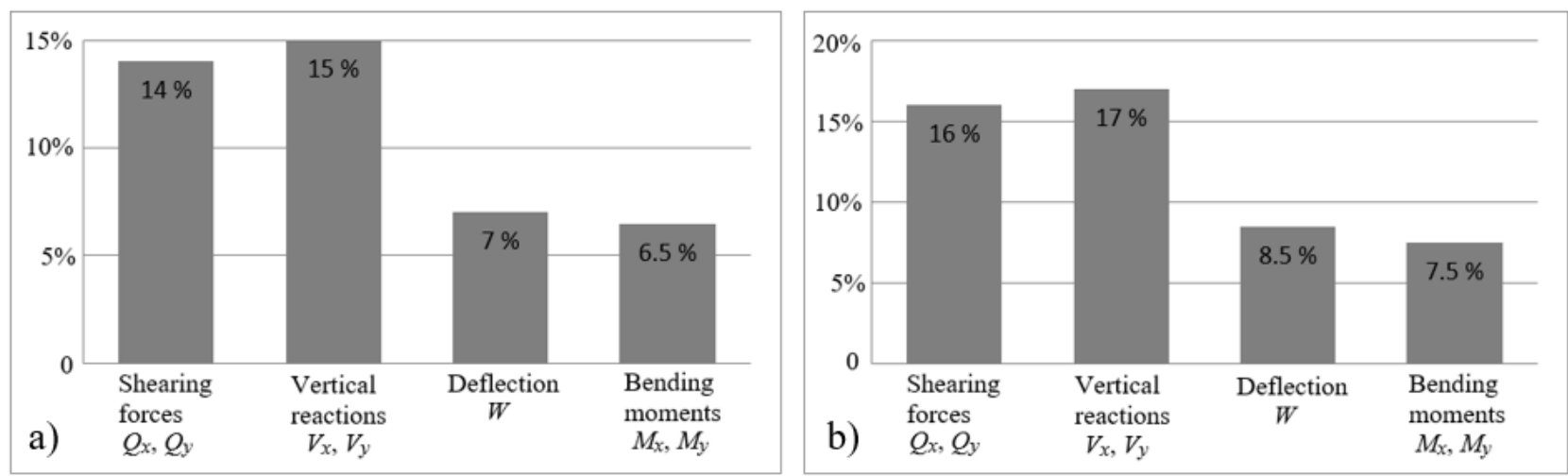

Fig.5. Deviation between the FEM and the real values for the uniform load distribution (a) and for the hydrostatic pressure (b).

\subsection{Analysis of methods for plate calculation}

In order to determine which of the methods is best for plate calculation and is closer to the real solution, a comparison of the deviations of all the methods is essential. The data of all the deviations for plates loaded by a distributed load are shown in Fig.6a, and for plates loaded by a hydrostatic pressure in Fig.6b.
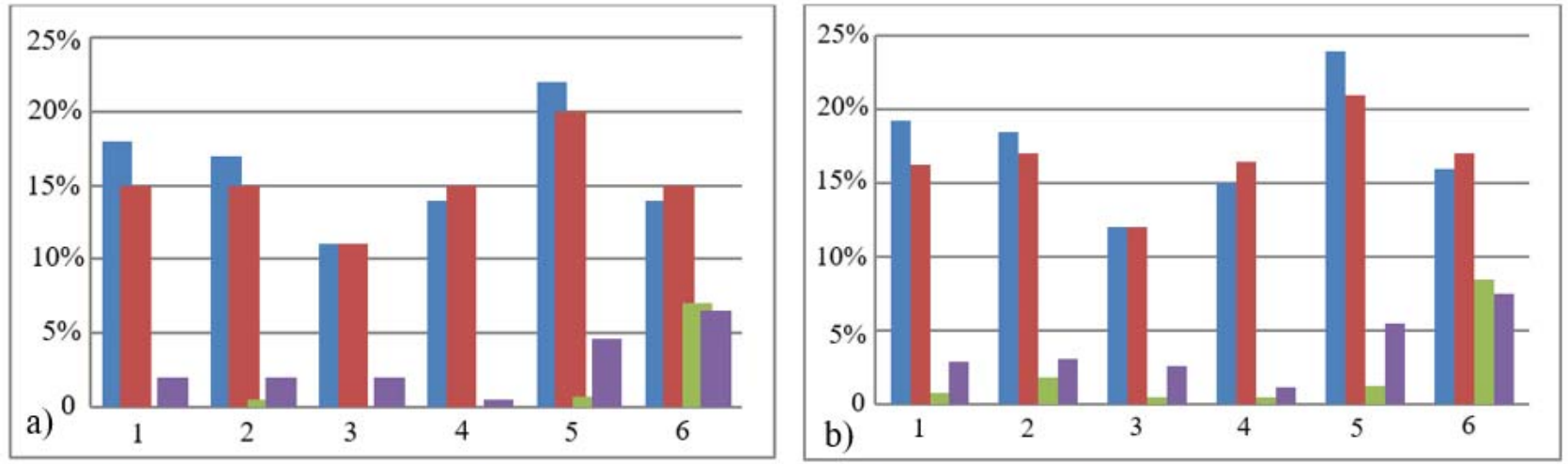

m Shearing forces Qx, Qy $=$ Vertical reations Vx, Vy $\equiv$ Deflection W $=$ Bending moments $\mathrm{Mx}, \mathrm{My}$

Fig.6. Deviation between all the methods and the real values for the uniform load distribution (a) and for the hydrostatic pressure (b). 1 - Navier' method, 2 - Levy solution, 3 - collocation method, 4 Kantorovits-Vlassov method, 5 - grid method, 6 - plate calculations by FEM.

Figure 6 demonstrates that the collocation, the Kantorovits-Vlassov and the Navier' methods have the smallest deviation from the real solution. The deviation for the deflection $w$ is varies from $0.5 \%$ to $0.8 \%$. Next in ranking are the grid and the Levy methods with deviations for the deflection $1.3 \%$ and $1.8 \%$, respectively. The FEM method has the largest deviation, accounting for a deflection of $8.5 \%$. In summary, all the methods except the FEM cannot be used for plate calculation loaded by the hydrostatic pressure taking account of the boundary conditions. If all the parameters will be taken into consideration, it can be concluded that the most suitable method for plate calculation loaded by the hydrostatic pressure is the FEM 
method. Taking into consideration that the FEM method does not depend on the boundary conditions, it suits best for the comparison of the proposed results.

\section{Calculation of plates with variable thickness}

To ensure a more optimal distribution of the stress, the plates can have variable thicknesses. These constructions are widely used in shipbuilding for most of the hull elements of the ships. In the plate calculations by variational methods it is necessary to determine the basic set functions for unknown variables, which satisfy the boundary conditions on the edge of the construction. The task is to develop the theory and methods for plates with variable thicknesses. For that purpose calculation algorithms are required, which can expand the area of plate calculation tasks improving the existing solutions.

In particular, the calculations are complex if the elements consist of variable thicknesses. The composite structures include beams, plates with stepwise changing stiffness as well as shells consisting of elements of various shapes. Composite structures are typically calculated by their decomposition into individual elements, where in each of them the stiffness and geometric characteristics change monotonously. For each obtained element, a solution must be known in advance. To ensure the neighboring conjugation sites on the displacements and the internal forces, a system of algebraic equations must be set up with unknowns. $N \cdot n$ is the order of the differential equation, and $N$ is the number of elements.

\subsection{Calculation of plates with variable thicknesses by generalized functions}

Taking into account the method of additional partial solutions for the properties of generalized functions, a method for calculation of rectangular plates with variable stiffness using generalized functions is developed. This method can be defined as a private case of a method of generalized solutions [41]. To solve this task taking into account some properties of generalized functions, it is necessary to develop a system of algebraic equations only with $n$ unknown.

Figure 7 shows a rectangular plate with variable thicknesses, freely supported at two opposite edges $y=0, y=b$ and two fixed edges $x=0, x=a$.
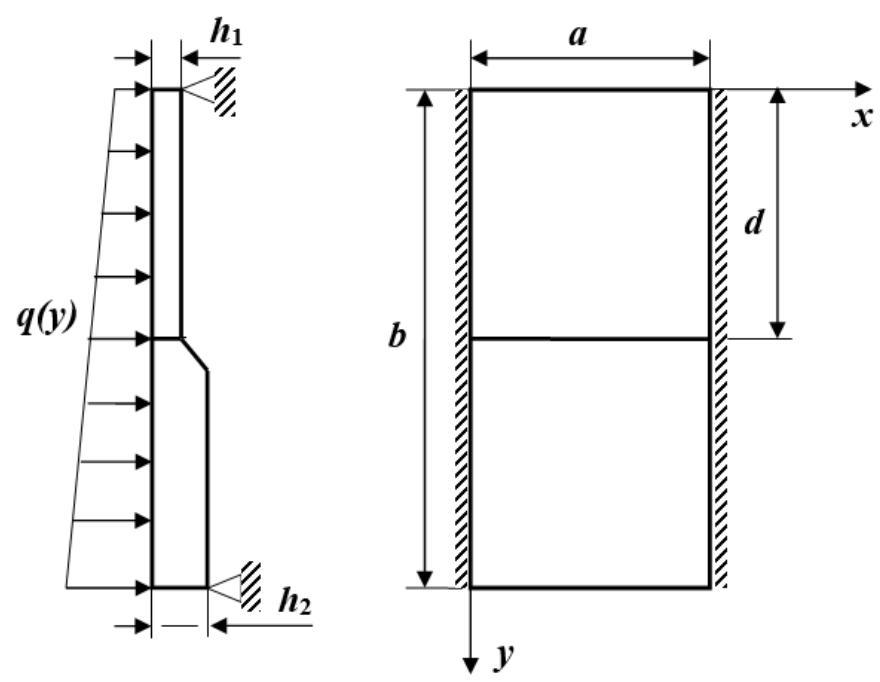

Fig.7. Rectangular plate with variable thicknesses with two opposite edges freely supported and two fixed edges.

The differential equation for the deflection surface $w(x, y)$ of the $i$-section of the plate is described by the biharmonic equation 


$$
\nabla^{2} \nabla^{2} w(x, y)=\frac{\partial^{4} w(x, y)}{\partial x^{4}}+2 \frac{\partial^{4} w(x, y)}{\partial x^{2} \partial y^{2}}+\frac{\partial^{4} w(x, y)}{\partial y^{4}}=\frac{q(x, y)}{D_{i}}
$$

where

$$
i=1,2, \ldots N
$$

The solution is in the form

$$
w(x, y)=Y(y) X(x)
$$

Taking into consideration that stiffness is changing unevenly only in one direction along the $y$ axis, the function $X(x)$ can be defined as the polynomial below

$$
X(x)=\left(1-\frac{x^{2}}{a^{2}}\right)^{2}
$$

and the deflection surface $w(x, y)$ will be

$$
w(x, y)=Y(y)\left(1-\frac{x^{2}}{a^{2}}\right)^{2}
$$

Equation (3.2) satisfies the boundary conditions $w(x, y)=0$ and $\frac{\partial w(x, y)}{\partial x}=0$ at these two sides $x=0, x=a$. The task is to determine $Y(y)$ in a form that would satisfy the boundary conditions on the sides $y=0, y=b$ and the equation of the deflection surface. The principle of virtual displacements will be used

$$
\int_{0}^{a}\left(\nabla^{2} \nabla^{2} w(x, y)-\frac{q}{D_{i}}\right) \cdot X(x) d x=\int_{0}^{a}\left(\nabla^{2} \nabla^{2} w(x, y)-\frac{q}{D_{i}}\right) \cdot\left(1-\frac{x^{2}}{a^{2}}\right)^{2} d x=0 .
$$

Let us place $w(x, y)$ from Eq.(3.4) to Eq.(3.5). Then we will obtain

$$
\int_{0}^{a}\left(\frac{24}{a^{4}} Y(y)+\frac{8}{a^{2}}\left(-1+3 \frac{x^{2}}{a^{2}}\right) Y^{\prime \prime}(y)+\left(1-\frac{x^{2}}{a^{2}}\right)^{2} Y^{I V}(y)-\frac{q}{D_{i}}\right) \cdot\left(1-\frac{x^{2}}{a^{2}}\right)^{2} d x=0 .
$$

After integration the results will be as follows

$$
\frac{8}{21} Y^{I V}(y)-\frac{16}{7 a^{2}} Y^{\prime \prime}(y)+\frac{24}{a^{4}} Y(y)=\frac{q}{D_{i}} .
$$

Since the structure consists of two sections (Fig.7), it is possible to consider the following auxiliary differential equation 


$$
\begin{aligned}
& \frac{8}{21} Y^{I V}(y)-\frac{16}{7 a^{2}} Y^{\prime \prime}(y)+\frac{24}{a^{4}} Y(y)=\frac{q}{D_{i}}+B_{0}(d) \cdot \delta(y-d)+ \\
& +B_{1}(d) \cdot \delta^{\prime}(y-d)+B_{2}(d) \cdot \delta^{\prime \prime}(y-d)+B_{3}(d) \cdot \delta^{\prime \prime \prime}(y-d)
\end{aligned}
$$

where $i=1,2$.

At this point, it is necessary to find a special solution of the differential Eq.(3.8) in this form

$$
\tilde{Y}_{r}(y)=\left\{[1-\eta(y-d)]\left[Z_{0}(y)+Z_{q}(y)\right]+\eta(y-d) V_{q}(y)\right\} .
$$

Consistently differentiating the expression (3.9) four times and taking into account the filtering property of the delta function, the following will be obtained:

$$
\begin{aligned}
& \tilde{Y}_{r}^{\prime}(y)=[1-\eta(y-d)]\left[Z_{0}^{\prime}(y)+Z_{q}^{\prime}(y)-V_{q}^{\prime}(y)\right]-\delta(y-d)\left[Z_{0}(y)+Z_{q}(y)-V_{q}(y)\right] \\
& \tilde{Y}_{r}^{\prime \prime}(y)=[1-\eta(y-d)]\left[Z_{0}^{\prime \prime}(y)+Z_{q}^{\prime \prime}(y)-V_{q}^{\prime \prime}(y)\right]+ \\
& -2 \delta(y-d)\left[Z_{0}^{\prime}(y)+Z_{q}^{\prime}(y)-V_{q}^{\prime}(y)\right]-\delta^{\prime}(y-d)\left[Z_{0}(y)+Z_{q}(y)-V_{q}(y)\right] \\
& \tilde{Y}_{r}^{\prime \prime \prime}(y)=[1-\eta(y-d)]\left[Z_{0}^{\prime \prime \prime}(y)+Z_{q}^{\prime \prime \prime}(y)-V_{q}^{\prime \prime \prime}(y)\right]-3 \delta(y-d)\left[Z_{0}^{\prime \prime}(y) y+Z_{q}^{\prime \prime}(y)-V_{q}^{\prime \prime}(y)\right]+ \\
& -3 \delta^{\prime}(y-d)\left[Z_{0}^{\prime}(y)+Z_{q}^{\prime}(y)-V_{q}^{\prime}(y)\right]-\delta^{\prime \prime}(y-d)\left[Z_{0}(y)+Z_{q}(y)-V_{q}(y)\right] \\
& \tilde{Y}_{r}^{I Y}(y)=[1-\eta(y-d)]\left[Z_{0}^{I Y}(y) y+Z_{q}^{I Y}(y)-V_{q}^{I Y}(x)\right]+ \\
& -4 \delta(y-d)\left[Z_{0}^{\prime \prime \prime}(y)+Z_{q}^{\prime \prime \prime}(y)-V_{q}^{\prime \prime \prime}(y)\right]-6 \delta^{\prime}(y-d)\left[Z_{0}^{\prime \prime}(y)+Z_{q}^{\prime \prime}(y)-V_{q}^{\prime \prime}(y)\right]+ \\
& -4 \delta^{\prime \prime}(y-d)\left[Z_{0}^{\prime}(y)+Z_{q}^{\prime}(y)-V_{q}^{\prime}(y)\right]-\delta^{\prime \prime \prime}(y-d)\left[Z_{0}(y)+Z_{q}(y)-V_{q}(y)\right]
\end{aligned}
$$

By substituting the values $\tilde{Y}_{r}^{I Y}(y), \tilde{Y}_{r}^{\prime \prime}(y)$ and $\tilde{Y}_{r}(y)$ in Eq.(3.8), and by comparing the coefficients of the delta-function and its derivatives on the left and right sides of Eq.(3.8), the unknown coefficients $B_{0}(d), B_{1}(d), B_{2}(d)$, and $B_{3}(d)$ for this equation must satisfy the relations, where four integration constants $C_{j}$ are included

$$
\begin{array}{ll}
B_{0}(d)=\left\{B_{3}^{\prime \prime \prime}(y)-\frac{12}{a^{2}} B_{3}^{\prime}(y)\right\} \mid y=d & B_{1}(d)=\left.\left\{4 B_{3}^{\prime \prime}(y)-\frac{6}{a^{2}} B_{3}(y)\right\}\right|_{y=d} ; \\
B_{2}(d)=\left.4 B_{3}^{\prime}(y)\right|_{y=d} ; & B_{3}(d)=\sum_{j=1}^{4} \frac{8}{21} C_{j}\left[V_{q}(y)-Z_{0 j}(y)-Z_{q}(y)\right] y=d,
\end{array}
$$

where $C_{j}$ integration constants determined from the boundary conditions, and $Z_{0 j}(y)$ are particular linearly independent solutions of the homogeneous differential Eq.(3.7):

From the homogeneous differential Eq.(3.7), the particular solutions $Z_{0_{j}}(y)$ will be 


$$
\begin{array}{ll}
Z_{01}(y)=\operatorname{ch} \frac{2.343 y}{a} \cos \frac{1.575 y}{a} ; & Z_{02}(y)=\operatorname{ch} \frac{2.343 y}{a} \sin \frac{1.575 y}{a} ; \\
Z_{03}(y)=\operatorname{sh} \frac{2.343 y}{a} \cos \frac{1.575 y}{a} ; & Z_{04}(y)=\operatorname{sh} \frac{2.343 y}{a} \sin \frac{1.575 y}{a} .
\end{array}
$$

Then the function

$$
\bar{Y}(y)=\sum_{j=1}^{4} C_{j}\left\{[1-\eta(y-d)] Z_{0 j}(x)\right\}+\left\{[1-\eta(y-d)] Z_{q}(x)+\eta(y-d) V_{q}(x)\right\},
$$

is the general solution of the differential Eq.(3.8).

It follows that the general solution of the initial differential Eq.(3.7) in the case of two sections is determined by the formula

$$
Y(y)=\bar{Y}(y)-\tilde{Y}_{r}(y),
$$

where $\tilde{Y}_{r}(y)$ is the special particular solution of the non-homogeneous differential Eq.(3.8) under the condition that the coefficients $B_{0}(d), B_{I}(d), B_{2}(d)$, and $B_{3}(d)$ satisfy the relations (3.11).

After completing the calculations, it is possible to obtain the main equation for the deflection by multiplying Eqs (3.4) and (3.24)

$$
w(x, y)=\left(\bar{Y}(y)-\tilde{Y}_{r}(y)\right)\left(1-\frac{x^{2}}{a^{2}}\right)^{2} .
$$

By using this equation the numerical results of the deflection of the plate can be obtained.

\subsection{Particular solution in the case of a uniformly distributed load}

A particular solution $\tilde{Y}_{r}(y)$ can be found by the method of variation of arbitrary constants. In this case we can obtain

$$
\begin{aligned}
& \tilde{Y}_{r}(y)=\frac{\eta(x-d)}{2}\left\{-Z_{01}(y)\left[\frac{a^{3} \cdot B_{0}(d)}{4 \sqrt{108}} Z_{02}(y)+\frac{a^{2} \cdot B_{1}(d)}{12} Z_{03}(y)+\right.\right. \\
& \left.-\frac{a \cdot B_{2}(d)}{\sqrt{6}} Z_{03}(y)-B_{3}(d) \cdot Z_{01}(y)\right]+Z_{02}(y)\left[\frac{a^{3} \cdot B_{0}(d)}{4 \sqrt{108}} Z_{03}(y)+\frac{a^{2} \cdot B_{1}(d)}{12} Z_{01}(y)+\right. \\
& \left.+\frac{a \cdot B_{2}(d)}{\sqrt{6}} Z_{04}(y)+B_{3}(d) \cdot Z_{02}(y)\right]-Z_{02}(y)\left[\frac{a^{3} B_{0}(d)}{4 \sqrt{108}} Z_{04}(y)-\frac{a^{2} \cdot B_{1}(d)}{12} Z_{01}(y)+\right. \\
& \left.\left.+\frac{a \cdot B_{2}(d)}{\sqrt{6}} Z_{03}(y)+B_{3}(d) \cdot Z_{02}(y)\right]\right\}
\end{aligned}
$$


where $Z_{01}(y), Z_{02}(y), Z_{03}(y)$ and $Z_{04}(y)$ are the particular solutions of the homogeneous differential Eq.(3.7), which are determined by the expressions (3.8) and the coefficients $B_{0}(d), B_{1}(d), B_{2}(d)$ and $B_{3}(d)$, that are

$$
\begin{aligned}
& B_{0}(d)=\frac{2 \sqrt{108}}{a^{3}}\left[C_{2} Z_{03}(d)-C_{1} Z_{04}(d)+C_{3} Z_{03}(d)+C_{4} Z_{04}(d)\right] \\
& B_{1}(d)=\frac{24}{a^{2}}\left[C_{2} Z_{01}(d)-C_{1} Z_{02}(d)+C_{3} Z_{02}(d)+C_{4} Z_{01}(d)\right] \\
& B_{2}(d)=\frac{\sqrt{6}}{a}\left[C_{1} Z_{03}(d)+C_{2} Z_{04}(d)+C_{3} Z_{04}(d)-C_{4} Z_{03}(d)\right] \\
& B_{3}(d)=C_{1}\left[Z_{01}(d)-Z_{02}(d)\right]+C_{2}\left[Z_{02}(d)-Z_{03}(d)\right]+ \\
& +C_{3}\left[Z_{03}(d)-Z_{04}(d)\right]+C_{4}\left[Z_{04}(d)-Z_{01}(d)\right]+\left(\frac{a^{4}}{36 D_{2}}+\frac{a^{4}}{36 D_{1}}\right) \frac{q}{4}
\end{aligned}
$$

Thus, the general solution of the non-homogeneous differential Eq.(3.1) can be found by the following formula, taking into account Eqs (3.13), (3.14) and (3.15)

$$
\begin{aligned}
& Y(y)=C_{1}[1-\eta(y-d)] Z_{01}(y)+C_{2}[1-\eta(y-d)] Z_{02}(y)+ \\
& +C_{3}[1-\eta(y-d)] Z_{03}(y)+C_{4}[1-\eta(y-d)] Z_{04}(y)+ \\
& +\left\{[1-\eta(y-d)] \frac{a^{4}}{36 D_{1}}+\eta(y-d) \frac{a^{4}}{36 D_{2}}\right\} \frac{q}{4}-\tilde{y}_{r}(x) .
\end{aligned}
$$

If the construction contains several sections with different stiffness characteristics, the general solution can be written as follows

where

$$
\begin{aligned}
& Y(y)=\sum_{l=1}^{\alpha} C_{1}\left\{\left[\eta\left(y-d_{l-1}\right)-\eta\left(y-d_{l}\right)\right] Z_{01}(y)+\right. \\
& C_{2}\left[\eta\left(y-d_{l-1}\right)-\eta\left(y-d_{l}\right)\right] Z_{02}(y)+C_{3}\left[\eta\left(y-d_{l-1}\right)-\eta\left(y-d_{l}\right)\right] Z_{03}(y)+ \\
& \left.+C_{4}\left[\eta\left(y-d_{l-1}\right)-\eta\left(y-d_{l}\right)\right]+\left[\eta\left(y-d_{l-1}\right)-\eta\left(y-d_{l}\right)\right] \frac{a^{4} q}{36 D_{l}}\right\}-\tilde{Y}_{r}(y)
\end{aligned}
$$

$d_{l-1}(l=1,2, \ldots, \alpha)$ are the coordinates of the points in which the stiffness characteristics of the construction are changed.

The particular solution $\tilde{Y}_{r}(y)$, which is included in Eq.(3.19), takes the form

$$
\tilde{Y}_{r}(y)=\sum_{l}^{\alpha-1} \tilde{Y}_{r l}(y)
$$


where $\tilde{Y}_{r l}(y)$ is the partial solution, which can be calculated by Eq.(3.16) with the replacement $d$ for $d_{l}$. Equation (3.19) shows that the unknown included in this formula are arbitrary constants $C_{1}, C_{2}, C_{3}$ and $C_{4}$ which can be calculated from the boundary conditions of the problem.

\subsection{Particular solution in the case of hydrostatic pressure}

Similarly, the particular solution $\widetilde{Y}_{r}(y)$ for the hydrostatic pressure can be found. In this case the differential Eq.(3.8) will be

$$
\begin{aligned}
& \frac{8}{21} Y^{I V}(y)-\frac{16}{7 a^{2}} Y^{\prime \prime}(y)+\frac{24}{a^{4}} Y(y)=\frac{q_{0} \cdot y}{D_{i} \cdot b}+B_{0}(d) \cdot \delta(y-d)+ \\
& +B_{1}(d) \cdot \delta^{\prime}(y-d)+B_{2}(d) \cdot \delta^{\prime \prime}(y-d)+B_{3}(d) \cdot \delta^{\prime \prime \prime}(y-d), \quad(i=1,2) .
\end{aligned}
$$

Similarly to the case of the uniformly distributed load, the particular solution $\tilde{Y}_{r}(y)$ for the hydrostatic pressure can be found by the method of variation of arbitrary constants. In this case it will be

$$
\begin{aligned}
& \tilde{Y}_{r}(y)=\frac{\eta(y-d)}{2}\left\{-Z_{01}(y)\left[\frac{a^{3} \cdot B_{0}(d)}{4 \sqrt{108}} Z_{02}(y)+\frac{a^{2} \cdot B_{1}(d)}{12} Z_{03}(y)+\right.\right. \\
& \left.-\frac{a \cdot B_{2}(d)}{\sqrt{6}} Z_{03}(y)-y \cdot B_{3}(d) \cdot Z_{01}(y)\right]+Z_{02}(y)\left[\frac{a^{3} \cdot B_{0}(d)}{4 \sqrt{108}} Z_{03}(y)+\frac{a^{2} \cdot B_{1}(d)}{12} Z_{01}(y)+\right. \\
& \left.+\frac{a \cdot B_{2}(d)}{\sqrt{6}} Z_{04}(y)+y \cdot B_{3}(d) \cdot Z_{02}(y)\right]-Z_{02}(y)\left[\frac{a^{3} B_{0}(d)}{4 \sqrt{108}} Z_{04}(y)-\frac{a^{2} \cdot B_{1}(d)}{12} Z_{01}(y)+\right. \\
& \left.\left.+\frac{a \cdot B_{2}(d)}{\sqrt{6}} Z_{03}(y)+y \cdot B_{3}(d) \cdot Z_{02}(y)\right]\right\}
\end{aligned}
$$

where $Z_{01}(y), Z_{02}(y), Z_{03}(y)$ and $Z_{04}(y)$ are the particular solutions of the homogeneous differential Eq.(3.7), which are determined by Eq.(3.12) and the coefficients $B_{0}(d), B_{l}(d), B_{2}(d)$ and $B_{3}(d)$ are

$$
\begin{aligned}
& B_{0}(d)=\frac{2 \sqrt{108}}{a^{3}}\left[C_{2} Z_{03}(d)-C_{1} Z_{04}(d)+C_{3} Z_{03}(d)+C_{4} Z_{04}(d)\right] \\
& B_{1}(d)=\frac{24}{a^{2}}\left[C_{2} Z_{01}(d)-C_{1} Z_{02}(d)+C_{3} Z_{02}(d)+C_{4} Z_{01}(d)\right] \\
& B_{2}(d)=\frac{\sqrt{6}}{a}\left[C_{1} Z_{03}(d)+C_{2} Z_{04}(d)+C_{3} Z_{04}(d)-C_{4} Z_{03}(d)\right] \\
& B_{3}(d)=C_{1}\left[Z_{01}(d)-Z_{02}(d)\right]+C_{2}\left[Z_{02}(d)-Z_{03}(d)\right]+C_{3}\left[Z_{03}(d)-Z_{04}(d)\right]+ \\
& +C_{4}\left[Z_{04}(d)-Z_{01}(d)\right]+\left(\frac{a^{4}}{72 D_{1}} \cdot \frac{d}{b}+\frac{a^{4}}{48 D_{2}}+\right) \frac{q_{0}}{4} .
\end{aligned}
$$


Thus, the general solution of the non-homogeneous differential Eq.(3.1) for the hydrostatic pressure, taking into account Eqs (3.13), (3.14) and (3.22), can be found by the next formula

$$
\begin{aligned}
& Y(y)=C_{1}[1-\eta(y-d)] Z_{01}(y)+C_{2}[1-\eta(y-d)] Z_{02}(y)+ \\
& +C_{3}[1-\eta(y-d)] Z_{03}(y)+C_{4}[1-\eta(y-d)] Z_{04}(y)+ \\
& +\left\{[1-\eta(y-d)] \frac{a^{4}}{72 D_{1}} \cdot \frac{d}{b}+\eta(y-d) \frac{a^{4}}{48 D_{2}}\right\} \frac{q_{0}}{4}-\tilde{Y}_{r}(y) .
\end{aligned}
$$

The obtained results can be generalized to any type of a composite construction of beams and plates. The number of arbitrary constants of integration will be equal to the order of the differential equations corresponding to the considered type of constructions. These constants are determined from the appropriate boundary conditions of the problem.

\subsection{Results of calculations}

In order to solve the equations and to obtain the numerical results of deflection the Matlab program is used. The results are presented by the values of deflection for all the cases of the calculation (Tab.1):

1) plate with constant thickness $(h=8 \mathrm{~mm})$ loaded by the uniformly distributed load,

2) plate with constant thickness $(h=8 \mathrm{~mm})$ loaded by the hydrostatic pressure,

3) plate with variable thicknesses $\left(h_{1}=6 \mathrm{~mm}, h_{2}=8 \mathrm{~mm}\right.$, Fig.7) loaded by the uniformly distributed load,

4) plate with variable thicknesses $\left(h_{1}=6 \mathrm{~mm}, h_{2}=8 \mathrm{~mm}\right.$, Fig.7) loaded by the hydrostatic pressure.

Table 1. Maximum deflections of the plates.

\begin{tabular}{|c|c|c|c|c|}
\hline Load, $\mathrm{kPa}$ & 6 & 12 & 18 & 24 \\
\hline Case of calculation & & & & \\
\hline 1 & 0.110 & 0.240 & 0.370 & 0.490 \\
\hline 2 & 0.022 & 0.049 & 0.072 & 0.099 \\
\hline 3 & 0.160 & 0.320 & 0.510 & 0.690 \\
\hline 4 & 0.031 & 0.062 & 0.093 & 0.133 \\
\hline
\end{tabular}

The total loads are equal to $6 \mathrm{kPa}, 12 \mathrm{kPa}, 18 \mathrm{kPa}$ and $24 \mathrm{kPa}$ for the uniformly distributed load. The same loads are used for the calculations under hydrostatic pressure. The calculations were made for plates with constant $(8 \mathrm{~mm})$ and variable $(6 \mathrm{~mm}$ and $8 \mathrm{~mm})$ thickness with dimensions of $180 \mathrm{~mm}$ in width and 400 $\mathrm{mm}$ in length loaded by the uniformly distributed load and hydrostatic pressure. The steel grade NVA with yield stress $235 \mathrm{~N} / \mathrm{mm}^{2}$ is used.

Deflections along all widths of the plate are presented in Fig. 8 for all four cases of loading. The figure and Tab.1 show that deflection of the plates with variable thickness is $40 \%$ and $34 \%$ more in comparison to the deflection of the plates with constant thickness for uniform load distribution and hydrostatic pressure accordingly. 

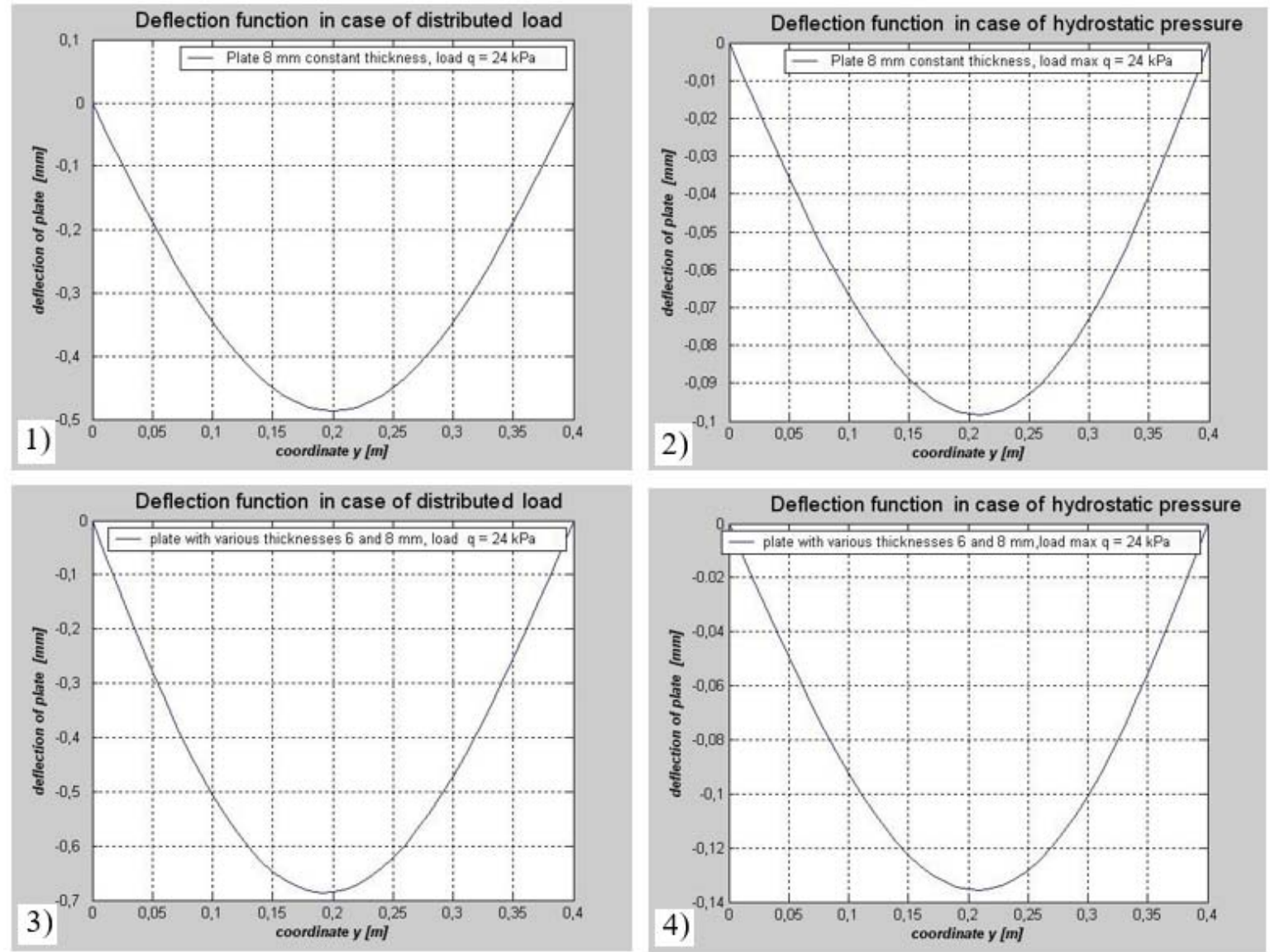

Fig.8. Curve of the plates deflection.

\section{Experiments and FEM analysis}

In order to check and prove that this method of plate calculation is correct and can be used, a series of experiments were made with the use of a tensile test machine with additional equipment. The basis of test components are shown in Fig.9. FEM calculations were also made.

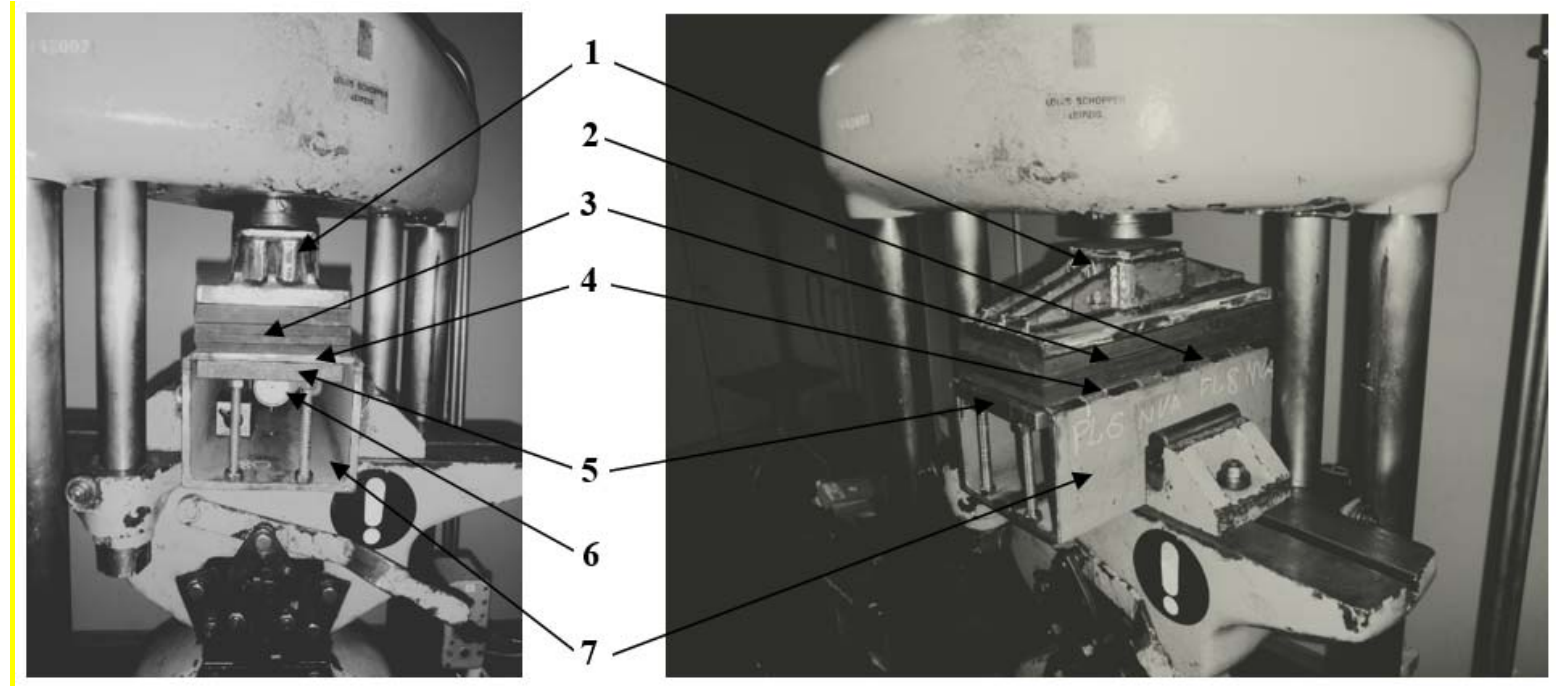

Fig.9. Tensile test machine with equipment. 1 -jig, 2 - plate with $h_{1}=6 \mathrm{~mm}, 3$ - rubber pie, 4 - plate with $h_{2}=8 \mathrm{~mm}, 5$ - freely-supported end, 6 - deflection indicator, 7 - U-channel. 
The experiments were carried out for two types of plates: a plate with variable thicknesses of $6 \mathrm{~mm}$ and $8 \mathrm{~mm}$, and a plate with a constant thickness of $8 \mathrm{~mm}$. The steel grade NVA with yield stress $235 \mathrm{~N} / \mathrm{mm}^{2}$ was used. The aim was to achieve the deflection magnitudes of the plate under a uniformly distributed load. The main challenge was to convert a single force to a distributed load. For this reason a special jig was designed and produced. Another aim was to imitate a distributed load on the whole surface of the plate. Therefore, a rubber pie was produced and connected to the jig. A medium soft rubber was used, which seems to suit perfectly for transferring a load on the whole surface of the plate.

The plate with variable thickness was fixed at two sides to the U-channel in order to imitate a real side of the plate. The U-channel with the plate was fixed to the table to avoid any movements during the pressure work. The deflection was measured by the clock indicator with an accuracy of $+/$ $0.01 \mathrm{~mm}$.

Table 2. Deflection $(\mathrm{mm})$ of the plate with constant thickness under an uniformly distributed load.

\begin{tabular}{|c|c|c|c|c|}
\hline \multicolumn{5}{|c|}{$8 \mathrm{~mm}$ plate with constant thickness } \\
\hline Attempt & Load 6 kPa & Load $12 \mathrm{kPa}$ & Load $18 \mathrm{kPa}$ & Load $24 \mathrm{kPa}$ \\
\hline 1 & 0.12 & 0.26 & 0.37 & 0.47 \\
\hline 2 & 0.14 & 0.27 & 0.37 & 0.48 \\
\hline 3 & 0.14 & 0.27 & 0.38 & 0.46 \\
\hline
\end{tabular}

In these experiments, three attempts of each load were made with twelve measurements of the deflection in order to achieve more accurate results. In the first experiment, a plate with a constant thickness of $8 \mathrm{~mm}$ was used. The dimensions of the plate were $180 \mathrm{~mm}$ in width and $400 \mathrm{~mm}$ in length. The plate was fixed at two sides and two sides were freely supported. The dimensions of the plate were chosen due to the limited area of the stand of the setup. The results of the experiments are presented in the Tab.2 and Tab.3.

Table 3. Deflection $(\mathrm{mm})$ of the plate with variable thicknesses under an uniformly distributed load.

\begin{tabular}{|c|c|c|c|c|}
\hline \multicolumn{5}{|c|}{$6-8 \mathrm{~mm}$ plate with variable thicknesses } \\
\hline Attempt & Load 6 kPa & Load $12 \mathrm{kPa}$ & Load $18 \mathrm{kPa}$ & Load 24 kPa \\
\hline 1 & 0.15 & 0.29 & 0.44 & 0.59 \\
\hline 2 & 0.15 & 0.30 & 0.44 & 0.58 \\
\hline 3 & 0.16 & 0.29 & 0.43 & 0.57 \\
\hline
\end{tabular}

FEM calculations were done by help of the Solid Works Cosmos program. In the calculation the same data were used as in the experiment and in the method of analytical calculations. Two calculations for the plate with variable thicknesses of 6 and $8 \mathrm{~mm}$ and for the plate with a constant thickness of $8 \mathrm{~mm}$ were made.

The results from all the calculations have to be compared. Figure 10 shows the deflections of the plate for all four considered cases. 

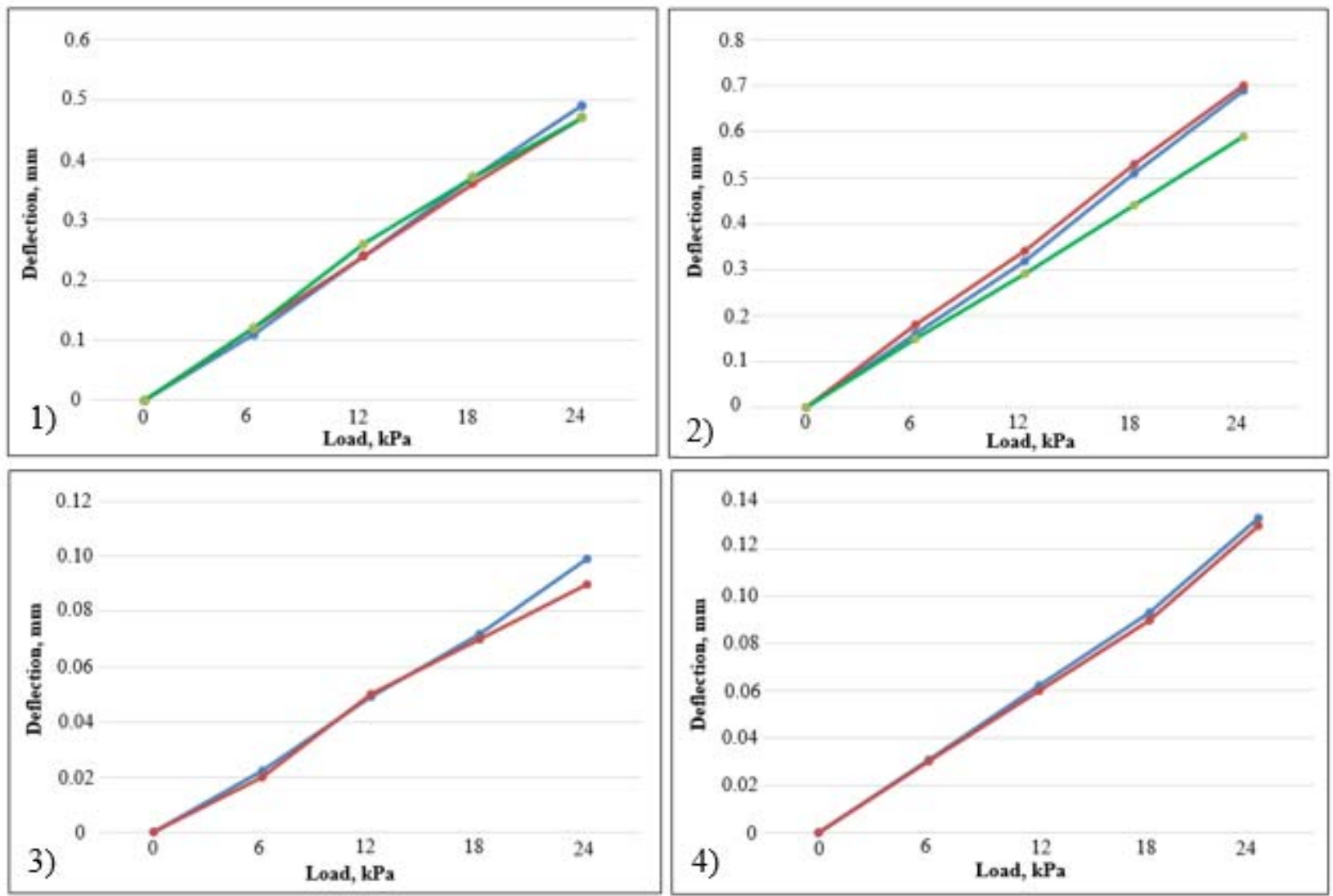

\section{$\sim$ calculation by proposed method \\ $\rightarrow$ calculation by FEM \\ $\longrightarrow$ experiment results}

Fig.10. Comparison of obtained results. 1 - plate with constant thickness and uniformly distributed load, 2 plate with variable thickness and uniformly distributed load, 3 - plate with constant thickness and hydrostatic pressure, 4 - plate with variable thickness and hydrostatic pressure.

Figure 10 shows that values calculated by proposed method are very close to the results obtained by FEM. Maximum deviation is about $11 \%$. Maximum deviation between experimental results and FEM calculation is $17 \%$. All this means that proposed method can be used for calculation of plates with variable thickness under uniformly loading or hydrostatic pressure.

\section{Conclusions}

An improved method for calculation of plates with variable thicknesses is proposed. This method is independent of the boundary conditions, which makes it different from other methods. A substantial advantage of the method of generalized function is that it can be compared to other methods used to solve the tasks of the theory of plates.

The achieved results show that the calculations with the new method are correct and they can be used for plates with variable thicknesses. By using Matlab software, the deflections of plates were found. The results of the proposed method are close to those of the FEM. The deviation is in the range of 0 to $11 \%$ compared with FEM calculations. 
The experiments with two type of plates were made. First, with an $8 \mathrm{~mm}$ plate of constant thickness and then, a $6 \mathrm{~mm}$ and $8 \mathrm{~mm}$ plate with variable thicknesses. The experimental results are close to the results of the proposed method. The maximum deviation is in the range of 0 to $17 \%$.

The proposed method make it possible to decrease the weight of the plates working under hydrostatic pressure by using variable thicknesses. The method can be used as a basis for the development of an algorithm for program calculations of plate thickness.

\section{Acknowledgements}

This research work was supported by innovative Manufacturing Engineering Systems Competence Centre IMECC (supported by Enterprise Estonia and co-financed by the European Union Regional Development Fund, project EU48685); Estonian Research Council grant PUT1300; the Estonian Centre of Excellence in Zero Energy and Resource Efficient Smart Buildings and Districts, ZEBE, grant TK146 funded by the European Regional Development Fund.

\section{Nomenclature}

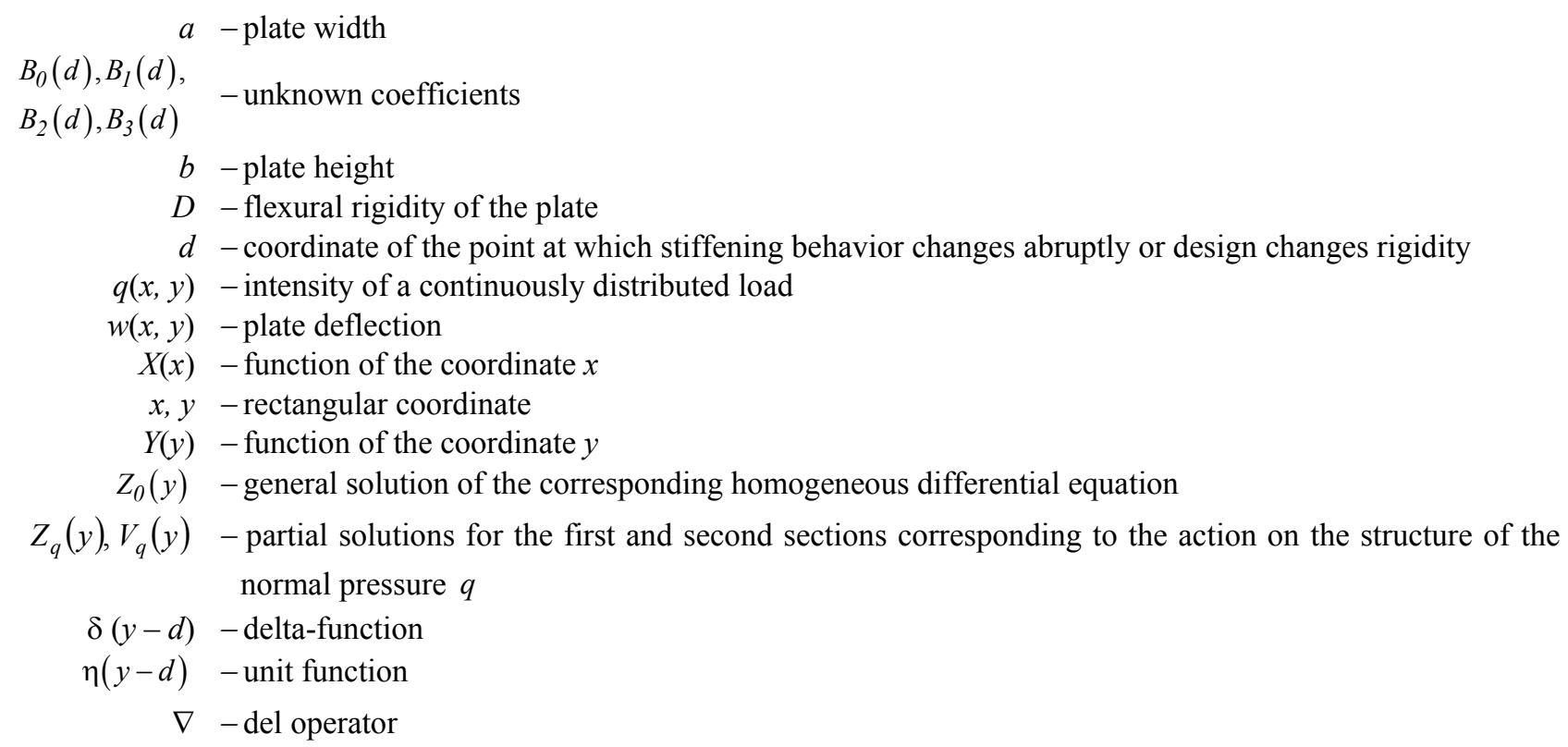

\section{References}

[1] Kanwal R.P. (1971): Linear Integral Equations Theory and Technique. - San Diego: Academic Press.

[2] Cheney W. and Kincaid D. (2008): Numerical Mathematics and Computing. - 6th Ed. Brooks/Cole, Independence.

[3] Agarwal R.P. and O'Regan D. (2009): Ordinary and partial differential equations with special functions, Fourier series and boundary value problems. - Ch. Lecture 14, Boundary Value Problems, Springer, New-York, pp.104108.

[4] Boyce W.E. and Di Prima R.C. (2009): Elementary Differential Equations and Boundary Value Problems. - 9th Ed. Wiley, Hoboken.

[5] Reut Z. (2005): Solution of ordinary differential equations by successive integration by parts. - International Journal of Mathematical Education in Science and Technology, vol.26, No.10, pp.589-597. 
[6] Koroljev V., Slepov V. and Sokolov E. (1980): Method of successive integration of differential equations of plates and shells. - Proceedings of Saint- Petersburg University of Architecture and Civil Engineering, Structural Mechanics, Saint-Petersburg, pp.45-51.

[7] Aryassov G. and Petritshenko A. (2009): Study of free vibration of ladder frames reinforced with plate. - Solid State Phenomena, Mechatronic Systems and Materials III, vols 147-149, pp.368-373.

[8] Zienkiewicz O. and Taylor R. (2005): Finite Element Method for Solid and Structural Mechanics. - 6th Ed. Butterworth-Heinemann.

[9] Vainberg D. and Vainberg E. (1970): Calculation of Plates. - Kiev: Budivelnic.

[10] Sapountzakis E. and Katsikadelis J. (2008): Elastic deformation of ribbed plates under static, transverseand inplane loading. - J. of Computers and Structures, vol.74, pp.571-581.

[11] Liew K. and Wang C. (2003): Rayleigh-Ritz method for general plate analysis. - Engineering Structures, vol.15, No.1, pp.55-60.

[12] Guryanov N. (1968): The effect of local loads on the Shallow spherical shell. - Investigations on the Theory of Plates and Shells, vol.6, pp.186-193.

[13] Shigalo J. (1975): Static of shells subjected to local loads. - Investigations on the Theory of Plates and Shells, vol.6, 62-91.

[14] Shigalo J. and Guryanov N. (1966): The effect of local loads on simply supported cylindrical shell. - Investigations on the Theory of Plates and Shells, vol.6, pp.42-55.

[15] Eek R. and Poverus L. (1967): Structural Mechanics. - Vol.2, Tallinn.

[16] Timoshenko S. (1990): Theory of Plates and Shells. - New-York: McCraw-Hill Bool Company, Inc..

[17] Mihailov B. (1980): Plates and shells with discontinuous parameters. - Saint-Petersburg.

[18] Sharkov O. and Zolotov I. (2011): Influence of the geometric properties of an external housing on its stress-strain state. - Russian Engineering Research, vol.31, No.4, pp.335-337, doi:10.3103/S1068798X11040228.

[19] Alfutov N., Zinovev P. and Popov B. (1984): Analysis of Multilayer Plates and Shells of Composite Materials. Moscow: Mashinostroenie.

[20] Gornostajev D., Aryassov G. and Penkov I. (2016): Calculation method for optimization of barge hull. International Review of Mechanical Engineering, vol.10, No.2, pp.115-124.

[21] Gornostajev D. (2014): Development of the Calculation Method of Barge Hull. - PhD Thesis, Tallinn University of Technology Press.

[22] Alexandrov A., Lamper R. and Suvernev V. (1985): Calculation of Structural Elements of Aircraft. Sandwich Plates and Shells. - Moscow: Mashinostroenie.

[23] Johnson R. (2010): Composite Structures of Steel and Concrete. - Vol.1: Beams, Slabs, Columns and Frames for Buildings, Blackwell Scientific Publishers, Oxford.

[24] Kristek V. and Skaloud M. (2001): Advanced Analysis and Design of Plated Structures. - Elsevier, Amsterdam and New York, and Academia (Publishing House of the Czechoslovak Academy of Sciences), Prague.

[25] Sharkov O., Koryagin S. and Velikanov N. (2016): Design Models for Shaping of Tooth Profile of External FineModule Ratchet Teeth. - IOP Conference Series: Materials Science and Engineering, Vol.124, 012165, doi:10.1088/1757-899X/124/1/012165.

[26] Leissa A. (2004): Buckling of Laminated Composite Plates and Shell Panels. - New York: American Society of Mechanical Engineers.

[27] Lewinski T. and Telega J. (2007): Plates, Laminates and Shells. Asymptotic Analysis and Homogenization. Singapore: World Scientific Publishing. 
[28] Reddy J. (2006): Analysis of Laminated Composite Plates and Shells. - New York, Chichester: John Wiley and Sons (A Wiley-Interscience Publication).

[29] Gilbert R. and Hackl K. (1995): Asymptotic Theories for Plates and Shells. - New York: John Wiley and Sons.

[30] Ivanyi M. and Skaloud M. (1995): Steel Plated Structures. - Wien: Springer-Verlag.

[31] Jawad M. (2004): Theory and Design of Plate and Shell Structures. - New York: Chapman and Hall.

[32] Sokolov E. (2004): Integration of Differential Equations in Shell Theory by the Method of Particular Solutions. Mathematical Modelling. Numerical Methods and Complex Programs. - St.-Peterburg: Proc. St.-Peterburg Civil University.

[33] Noor A., Burton W. and Bert C. (1996): Computational models for sandwich panels and shells. - Appl. Mech. Rev., vol.49, No.3.

[34] Mac Neal R. (1994): Finite elements: Their Design and Performance. - New York: Marcel Decker.

[35] Gould P. (1999): Analysis of Plates and Shells. Prentice Hall. - New Jersey: Upper Saddle River.

[36] Rvatsjov V., Kurpa L., Sklepus N. and Ucisvilli L. (1973): R-function method in tasks of deflection and vibrations in plates with complex shapes. - Kiev.

[37] Hutchinson J. (1991): Analysis of plates and shells by boundary collocations. - In: Boundary Element Analysis of Plates and Shells, Springer-Verlag, Berlin, pp.341-368.

[38] Vasiljev V. (1998): Classical theory of plates: historical perspective and state-of-the-art. - Mechanics of Solids, vol.33, No.3, pp.35-45.

[39] Rvatsjov V. and Kurpa L. (1987): R-Functions in Plate Theory. Kiev.

[40] Ventsel E. (2007): An indirect boundary element method for plate bending analysis. - Int. J. Numer. Meth. Eng., vol.9, pp.1597-1611.

[41] Aryassov G. and Zhigailov S. (2013): Optimal design of system of cross-beams. - Solid State Phenomena, Mechatronic Systems and Materials IY, vol.198, pp.675-680.

Received: July 17, 2017

Revised: June, 14, 2018 